\title{
Central corneal thickness in subjects with glaucoma and in normal individuals (with or without pseudoexfoliation syndrome)
}

This article was published in the following Dove Press journal:

Clinical Ophthalmology

29 September 2009

Number of times this article has been viewed

\author{
Georgios Kitsos \\ Christos Gartzios \\ loannis Asproudis \\ Eleni Bagli \\ Ophthalmology Department, \\ University of Ioannina, loannina, \\ Greece
}

Correspondence: Christos Gartzios Department of Opthalmology, Stavrakiou 84 , loannina 45500 , Greece

Tel +306945546228

$\mathrm{Fax}+302651020655$

Email gartziosc@yahoo.gr
Objective: The evaluation of central corneal thickness (CCT) in subjects with pesudoexfoliation glaucoma (PEXG), primary open-angle glaucoma (POAG), and in normotensive individuals with or without pseudoexfoliation syndrome (PXS).

Study design/patients and methods: CCT was evaluated with ultrasound pachymetry in a total of 179 individuals: 32 had bilateral PEXG, 55 had bilateral POAG, 35 had PXS, and 57 were healthy individuals without PXS.

Results: CCT in PEXG eyes $(526.00 \pm 34.30 \mu \mathrm{m})$ was significantly thinner compared to POAG eyes $(549.36 \pm 39.3 \mu \mathrm{m})(P=0.027)$ and normal control eyes with $(550.64 \pm 39.0 \mu \mathrm{m})$ or without PXS $(547.36 \pm 33.1 \mu \mathrm{m}),(P=0.039$ and 0.048 respectively $)$. No statistically significant difference was found comparing CCT values of POAG eyes to control group eyes.

Conclusion: The evaluation of CCT is necessary in all patients with glaucoma and especially in those with PEXG due to the thinner cornea and the risk of underestimation of intraocular pressure.

Keywords: CCT, pesudoexfoliation syndrome, glaucoma

\section{Introduction}

In routine clinical practice, intraocular pressure (IOP) is one of the most important parameters in the detection and monitoring of glaucoma. Goldmann applanation tonometry (GAT) has become the international "gold standard" for IOP measurements. The effect of central corneal thickness (CCT) on IOP readings using GAT has become a topic of much interest. ${ }^{1-7}$ A thick cornea would overestimate IOP and a thin one would underestimate it. Moreover the Ocular Hypertension Treatment Study (OHTS) designated CCT as a strong predictor for the development of primary open-angle glaucoma (POAG) in patients with ocular hypertension (OHT) ${ }^{8}$ Although the relationship between CCT and stage of glaucoma damage seems to be related to IOP measuring error, recent studies recognize CCT as an intrinsic ocular factor in the pathogenesis and progression of glaucoma. ${ }^{9-11}$

Being aware of these evidence, CCT assessment has been extensively studied in patients with POAG and has been shown to be thinner ${ }^{12-14}$ or similar4, ${ }^{15-19}$ to control individuals.

Pseudoexfoliation glaucoma (PEXG) is generally accepted as being more unpredictable and resistant to the treatment (surgical or medical) compared to POAG. Furthermore, the conversion of hypertensives with pseudoexfoliation syndrome (PXS) to glaucoma has been found to be more common than conversion of ocular hypertensives 
without PXS.20 In that way, evaluation of CCT in subjects with PXS or PEXG could be essential for early diagnosis and suitable management of the glaucoma. However, there are very few studies conducted in a small number of patients in the literature measuring the CCT in this type of glaucoma and their results are controversial. ${ }^{4,6,12,13,15,16,21,22}$

The aim of this study is the evaluation of central CCT in patients with PEXG, POAG, and in normotensive individuals with or without PXS.

\section{Patients and methods}

A total of 179 subjects (179 eyes), all Caucasians, were included in this cross-sectional study by a single observer. All subjects where examined in University Eye Clinic of Ioannina, within the context of routine examinations at the Glaucoma department between September 2007 and March 2008. Thirty-two subjects (32 eyes) had bilateral PEXG and 55 subjects (55 eyes) had bilateral POAG. The type of glaucoma had been diagnosed in previous appointments. Ninety two individuals (92 eyes) without glaucoma or OHT were also included in the study, comprising the control group. Thirty-five subjects (35 eyes) had bilateral PXS and 57 individuals (57 eyes) were normal without PXS.

All eyes underwent a full ophthalmologic examination, including applanation tonometry, gonioscopy, fundoscopy, and repeated Humphrey 24-2 static threshold perimetry (at least three examinations).

Data such as age, gender, ocular and medical history, number, type, and duration of antiglaucomatous medical therapy were also recorded.

Inclusion criteria for subjects with POAG were: untreated IOP more than $22 \mathrm{mmHg}$ on at least two diurnal curves, open-normal angle, glaucomatous optic disc and at least three Humphrey visual field tests with glaucomatous defects. Inclusion criteria for subjects with PEXG were: untreated IOP more than $22 \mathrm{mmHg}$, open angle, glaucomatous optic disc, at least three Humphrey visual field tests with glaucomatous defects and presence of typical pseudoexfoliative material at the anterior lens capsule and/or at the pupillary margin. On the day of examination, IOP measured by GAT was less than $21 \mathrm{mmHg}$ in all individuals with glaucoma.

Control group included subjects with IOP less than $21 \mathrm{mmHg}$, at three different successive measurements, open angle, normal optic disc, and Humphrey visual field tests and no family history of glaucoma. The control group was also evaluated for the presence of pseudoexfoliation syndrome (presence of typical pseudoexfoliative material at the anterior lens capsule and/or at the pupillary margin).
Subjects with ocular disease other than glaucoma, contact lens users and patients that had undergone ocular surgery or laser photocoagulation treatment were excluded. Also, subjects with myopia or hypermetropia greater then $3 \mathrm{D}$ or astigmatism more than $1 \mathrm{D}$ that could potentially effect our measurements were not included.

In all understudy groups, IOP was measured using calibrated GAT. Two measurements were made. If the two readings were within $2 \mathrm{mmHg}$, the mean of the two measurements was used. If the two readings were separated by more than $2 \mathrm{mmHg}$, a third measurement was made and the median became the recorded IOP.

The estimation of optic disc cupping (vertical C/D ratio) and retinal nerve fiber layer (RNFL)-average thickness (Avg. Thick.) was made with optical coherence tomography (Zeiss Stratus; Carl Zeiss, Inc., Valhalla, NY, USA).

Ultrasound pachymetry (Pacline; OPTIKON 2000 S.p.A, Rome, Italy) was used to obtain morning measurements, at least two hours after awakening, of central corneal thickness by a single observer. Repeated sets of five readings at the center of the cornea were taken, after a drop of anesthetic, until the standard deviation for the five readings were $5 \mu \mathrm{m}$ or less. Pachymetry always precedes applanation tonometry. One eye was randomly selected from each patient and analyzed in the study.

The measurements in each glaucoma type subgroup were compared against the age and sex matched control group.

Statistical analysis was performed with SPSS (v.15 for Windows XP; SPSS Inc., Chicago, IL, USA). One-way analysis of variance, Student's $t$-test and Chi squared $\left(\chi^{2}\right)$ test were used. One-way analysis of variance (ANOVA) using the Tukey post-hoc test was used to identify which groups (PXEG, POAG, control with or without PXS) differed from each other. We considered values of $P<0.05$ to be statistically significant.

\section{Results}

Eighty-seven subjects with glaucoma (32 with PEXG, 55 with POAG) and 92 control subjects (35 with PXS, 57 without PXS) met the inclusion criteria of the study. The demographic characteristics of each group are summarized in Table 1. All groups are similar in age and gender. Mean IOP measurements of subjects with glaucoma (POAG, PEXG) and of control group were below $21 \mathrm{mmHg}$, thus avoiding false measurements due to corneal edema (Table 1). PEXG individuals had statistically significant more severe glaucomatous optic nerve damage, as this is revealed by $\mathrm{C} / \mathrm{D}$ ratio and RNFL thickness, compared to subjects with POAG (Table 1; Student's $t$-test, $P=0.03$ and 0.01 , respectively). 
As far as the glaucoma subgroups are concerned, the study showed that they did not vary in type of topical antiglaucoma medication treatment. Topical carbonic anhydrase inhibitors and prostaglandine analogues were given to a similar percentage of patients in the two glaucoma subgroups. Also, the duration of topical antiglaucoma treatment was similar in both glaucoma subgroups.

Regarding CCT, our results showed the following mean measurements in the groups under study: $526.00 \pm 34.30 \mu \mathrm{m}$ in eyes with PEXG, $549.36 \pm 39.3 \mu \mathrm{m}$ in eyes with POAG, $550.64 \pm 39.0 \mu \mathrm{m}$ in the control group with PXS, and $547.36 \pm 33.1$ in the control group without PXS (Table 2, Figure 1). No statistically significant difference was found when comparing CCT values of subjects with POAG to control group with or without PXS (one-way ANOVA test, $P=0.999$ and 0.991 , respectively). However, subjects with PEXG had statistically significant thinner cornea compared to all the other groups (one-way ANOVA test: POAG group, $P=0.027$; control group with PXS, $P=0.039$; and control without PXS, $P=0.048$ ). Finally CCT was similar between control groups with or without pseudoexfoliation (one-way ANOVA test, $P=0.992$ ).

\section{Discussion}

It is well known that CCT affects IOP measurements using Goldmann applanation tonometry. ${ }^{1-7}$ Furthermore, OHTS demonstrated that CCT is also an important and independent risk factor for progression to initial glaucoma damage among persons with ocular hypertension. This could be attributed either to the fact that thinner corneas give lower measured IOP levels and these eyes may be subjected to less aggressive IOP-lowering therapy, or thinner corneas may be a risk factor due to an association with the response of the corneoscleral shell and ocular vasculature to IOP-induced stress.

In our study, CCT in POAG eyes did not have any significant difference compared to normal eyes. Our results are in concordance with many published studies. ${ }^{4,15-19,22}$ Ventura and colleagues, ${ }^{16}$ Herndon and colleagues, ${ }^{6}$ and Copt and colleagues ${ }^{19}$ compared CCT in patients suffering from POAG and in normal people and concluded that CCT does not differ significantly in these two groups. Although the small sample sizes of these studies could be an issue, Shah and colleagues, ${ }^{4}$ Jonas and colleagues, ${ }^{18}$ and Lee and colleagues ${ }^{17}$ came to the same conclusion when the CCT of 335, 215, and 343 eyes with POAG, respectively, were compared to normal eyes. On the contrary, according to other reports, CCT in POAG patients was found to be thinner compared to normal individuals. ${ }^{12-14}$ This difference could be attributed to the different ethnical make-up of the study populations. ${ }^{12}$ Investigators have shown that CCT is thinner in African Americans compared to Asians and Caucasians. ${ }^{23-25}$ Furthermore, more African Americans are likely to be in the POAG group since the incidence of POAG is higher in this race. However these demographic data are

Table I Characteristics for each of the investigated groups

\begin{tabular}{|c|c|c|c|c|c|c|}
\hline & PEXG & POAG & $P$ & CTL-PXS & CTL & $P$ \\
\hline Age (years) & $71.2 \pm 6.7$ & $69.5 \pm 8.5$ & $>0.05$ & $71.4 \pm 8.2$ & $69.4 \pm 11.1$ & $>0.05$ \\
\hline Sex *Male & 15 & 27 & $>0.05$ & 17 & 28 & $>0.05$ \\
\hline Female & 17 & 28 & & 18 & 29 & \\
\hline IOP (mm Hg) & $15.7 \pm 6.3$ & $15.2 \pm 2.5$ & $>0.05$ & $13.9 \pm 3.14$ & $14.2 \pm 2.3$ & $>0.05$ \\
\hline$C / D$ ratio $^{\dagger}$ & $0.60 \pm 0.22$ & $0.45 \pm 0.19$ & 0.03 & $0.28 \pm 0.1$ & $0.25 \pm 0.075$ & $>0.05$ \\
\hline $\begin{array}{l}\text { Avg RNFL thickness } \\
(\mu \mathrm{m})^{\dagger \dagger}\end{array}$ & $66.1 \pm 15.2$ & $75.8 \pm 13.6$ & 0.01 & $99.4 \pm 10.2$ & $100.1 \pm 10.2$ & $>0.05$ \\
\hline $\begin{array}{l}\text { Duration of medication } \\
\text { (months) }\end{array}$ & $36.2 \pm 6.2$ & $36.4 \pm 6.3$ & $>0.05$ & - & - & - \\
\hline Number of medications & $1.43 \pm 0.07$ & $1.54 \pm 0.08$ & $>0.05$ & - & - & - \\
\hline (Prost.or/and CAls)* & $22 / 32$ (78.2\%) & $43 / 55(68.8 \%)$ & $>0.05$ & - & - & - \\
\hline Prost & $16 / 22$ & $31 / 43$ & $>0.05$ & & & \\
\hline CAls & $3 / 22$ & $6 / 43$ & $>0.05$ & & & \\
\hline Prost and CAls & $3 / 22$ & $6 / 43$ & $>0.05$ & & & \\
\hline $\mathrm{N}$ & 32 & 55 & & 35 & 57 & \\
\hline
\end{tabular}

Notes: ${ }^{\dagger}$ Comparison of PEXG to POAG; statistically significant at $P=0.03(<0.05)$ Student's $t$-test; ${ }^{+\dagger}$ Comparison of PEXG to POAG: $P=0.01(<0.05)$ Student's $t$-test; *Chi squared test.

Abbreviations: POAG, primary open-angle glaucoma; PEXG, pseudoexfoliation glaucoma; CTL-PXS, Control group with pseudoexfoliation syndrome; CTL, control group without pseudoexfoliation syndrome; C/D ratio, cup to disc ratio; Prost, prostaglandin analogues; CAls: carbonic anhydrase inhibitors. 
Table 2 Central corneal thickness in glaucoma subgroups and control group

\begin{tabular}{lllll}
\hline & PEXG & POAG & CTL-PXS & CTL \\
\hline CCT & $526.00 \pm 34.30^{\dagger}$ & $549.36 \pm 39.3$ & $550.64 \pm 39.0$ & $547.36 \pm 33.1$ \\
$(\mu \mathrm{m})$ & & & & \\
\hline
\end{tabular}

Note: SStatistically significant at $P=0.021(<0.05)$ ANOVA test.

Abbreviations: $\mathrm{CCT}$, central corneal thickness; POAG, primary open-angle glaucoma; PEXG, pseudoexfoliation glaucoma; CTL-PXS, control group with pseudoexfoliation syndrome; CTL, control group without pseudoexfoliation syndrome.

not presented in most of the above reports. Alternatively, it has been shown ${ }^{26}$ that CCT is a powerful clinical factor in determining glaucoma severity at the initial examination by a glaucoma specialist. POAG patients with lower CCT had worse baseline visual fields than patients presenting thicker CCT. ${ }^{18,27-29}$ In the light of this evidence, differences of the glaucoma stage between POAG groups among the various studies could be at least partially responsible for the disparate conclusions.

Concerning CCT in subjects with PEXG and PXS, references in the literature are limited and to a certain degree ambiguous. Previous studies have shown that CCT in patients with PEXG is thinner compared to the control individuals. ${ }^{12,13,15}$ More specifically, studies by Aghaian and colleagues ${ }^{12}$ using an ultrasonic pachymeter have shown that the CCT in patients with PEXG is statistically significant thinner compared to normal individuals. Bechmann and colleagues ${ }^{13}$ reached the same conclusion with optical coherence tomography. However, according to other investigators, although CCT was lower in PEXG compared to control subjects this difference was not statistically significant. 4,6,16,21,22 The basis for these discordant results is unclear. The small number of included subjects could be a limitation at these studies and could also account for the controversial results.

In their study, Hepsen and colleagues ${ }^{30}$ reported that the mean CCT $(546.4 \mu \mathrm{m})$ in eyes with PXS was not significantly different compared to control eyes $(542.9 \mu \mathrm{m})$ using optical pachymetry. When those eyes were subcategorized, according to IOP, the CCT was significantly thinner in normotensive PXS eyes compared to those with hypertensive PXS and PEXG eyes. However, hypertensive PXS and PEXG eyes had an IOP higher than $21 \mathrm{mmHg}$ and this could affect the CCT measurements, considering also the fact that corneal endothelial density is decreased in PXS eyes. ${ }^{21}$

We included a higher number of PEXG subjects in our study compared to the reports above. We further subcategorized the control individuals depending on the presence or not of PXS. Our results showed that PEXG eyes had thinner CCT compared to POAG and normal eyes (with or without PXS).
Kniestedt and colleagues ${ }^{25}$ compared PEXG to POAG patients and came to the same conclusion although the POAG group in that study was multiracial. Other reports did not show a difference in CCT between PEXG and POAG subjects, but either the number of PEXG eyes was small ${ }^{4,16,22}$ or the POAG group was multiracial. ${ }^{12}$ Differences in the usage of carbonic anhydrase inhibitors and prostaglandin analogues as antiglaucoma medications has been shown to affect CCT readings, ${ }^{6,31-33}$ but could not explain our result since there was no variation between the two glaucoma groups concerning their treatment.

A question that arises from our findings is why eyes with PEXG present a thinner cornea compared to those with POAG and PXS.

In our study, subjects with PEXG were recruited from the hospital where they presented with more advanced, glaucoma alterations and thinner CCT compared to subjects with POAG. This fact could be attributed to a delay in the diagnosis or to deficient treatment of subjects with PEXG by their ophthalmologists prior to their visit to our glaucoma department. Indeed, the systematic underestimation of IOP in people with undiagnosed PEXG and thinner corneas, taking into account the nature of PEXG and PXS as having major variations in IOP, deprived the ophthalmologist of the diagnostic value of the most important risk factor (IOP) for the development of glaucoma as well as of the possibility of achieving "target pressure". Because of this, subjects developed more advanced glaucomatous alterations which led them to a more specialized glaucoma unit in our geographical area in order to be re-evaluated (glaucoma department, University Hospital). This is further confirmed by previous studies, where a significant negative correlation was found between CCT and C/D ratio, ${ }^{25,26}$ which indicates that patients with thin corneas are more likely to be found in an advance stage of glaucoma at their first presentation. In this way, subjects with advanced PEXG and thin CCT probably enriched our sample and their comparison with less advanced POAG subjects and PXS individuals gave us this statistical result.

If we take for granted that the CCT is an independent biological risk factor, then the co-existence of PXS and thin cornea may increase the risk for development of PEXG. There are studies that indicate a possible biological link between aspects of the front of the eye that can be measured, such as thickness or material properties of the cornea, and the structure, deformability, or physiology of the optic disc, lamina cibrosa, and peripapillary sclera. Findings by $\mathrm{OHTS}^{8}$ and other studies ${ }^{9-11}$ have generated an enormous interest 


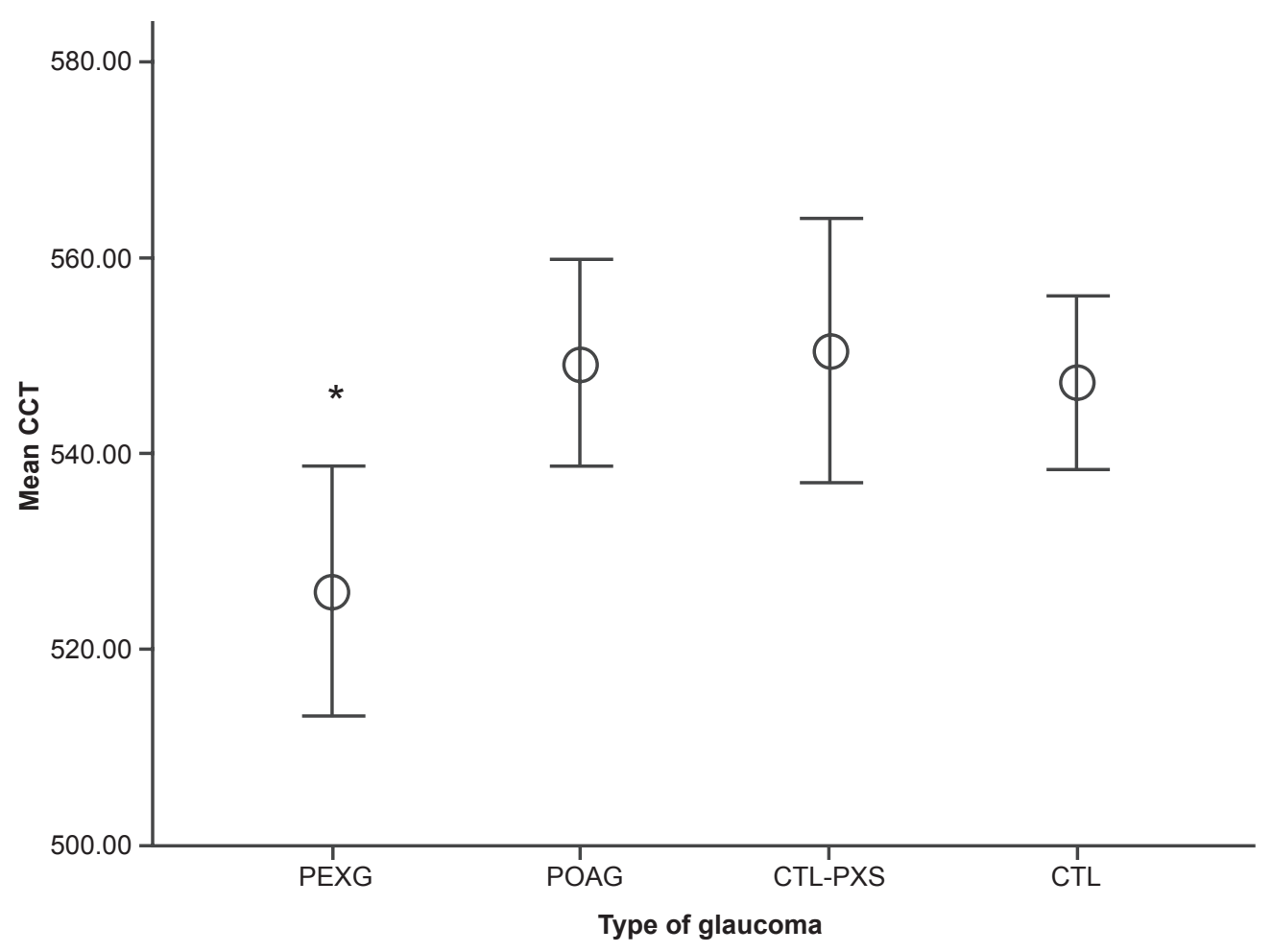

Error bars: $95 \% \mathrm{Cl}$

Figure I Central corneal thickness (CCT) in glaucoma subgroups and control group. The CCT values were measured with the PACLINE OPTICON 2000 S.P.A.The mean CCT with error bars in the different glaucoma and control groups are plotted.

Note: *Statistically significant at $P=0.021(<0.05)$ ANOVA test.

Abbreviations: POAG, primary open-angle glaucoma; PEXG, pseudoexfoliation glaucoma; CTL-PXS, control group with pseudoexfoliation syndrome; CTL, control group without pseudoexfoliation syndrome; $\mathrm{Cl}$, confidence interval.

in the material properties of the ocular coats of the eye and the likelihood that they may be an underlying factor in some aspects of the genetic susceptibility to glaucoma. Leske and colleagues ${ }^{9}$ used confocal scanning laser opthalmoscopy to measure the movement of lamina cibrosa after profound IOP lowering. They found that the lamina moved forward more in patients with thin corneas than in those with thick corneas. Pakravan and colleagues ${ }^{10}$ reported that CCT was linked to disc size and thicker corneas were associated with smaller optic disc. Finally, Toh and colleagues ${ }^{11}$ showed that CCT is among the most heritable aspects of ocular structure. There may be a biological link between the anterior chamber of the eye (such as CCT) and the peripapillary scleral tissue as well as the properties of the lamina cibrosa. If such a biological link is real then the co-existence of thin CCT with another predisposing factor for the development of glaucoma such as PXS increases the risk of glaucoma in those individuals.

Finally, we showed that CCT was not affected by the presence of pseudoexfoliation in individuals without glaucoma and our result is in agreement with previous studies. ${ }^{34,35} \mathrm{On}$ the contrary, Inoue and colleagues ${ }^{21}$ reported thinner corneas in individuals with PXS compared to the control. Finally, Puska and colleagues ${ }^{36}$ reported that CCT in eyes with PXS was thicker than the CCT found on the other eye of the same patient that did not have PXS.

In conclusion, our report demonstrates that $\mathrm{CCT}$ in subjects with PEXG was statistically significant thinner compared to the CCT of the rest understudy group and specially compared to CCT of individuals with PXS. This may be a statistic result but it could also indicate a possible independent biological role of CCT as a risk factor for the development of glaucoma in subjects with PXS. In that way CCT must be assessed in patients with PXS in order to avoid the underestimation of IOP (in cases with thin cornea). In addition, CCT must be determined in all glaucoma patients in order to achieve "target pressure", especially in patients with PEXG due to the nature of the disease (major variations of IOP).

\section{Disclosures}

The authors report no conflicts of interest in this work. 


\section{References}

1. Goldmann H, Schmidt T. Ueber applanationtonometrie. Ophthalmologica. 1957;134:221-241.

2. Ehlers N, Bramsen T, Sperling S. Applanation tonometry and central corneal thickness. Acta Ophthalmol (Copenh). 1975;53:34-43.

3. Doughty MJ, Zaman ML. Human corneal thickness and its impact on intraocular pressure measures: a review and metaanalysis approach. Surv Ophthalmol. 2000;44:367-408.

4. Shah S, Chatterjee A, Mathai M, et al. Relationship between corneal thickness and measured intraocular pressure in a general ophthalmology clinic. Ophthalmology. 1999;106:2154-2160.

5. Brandt JD, Beiser JA, Kass MA, et al. Central corneal thickness in the Ocular Hypertension Treatment Study (OHTS). Ophthalmology. 2001;108:1779-1788.

6. Herndon LW, Choudhri SA, Cox T, et al. Central corneal thickness in normal, glaucomatous, and ocular hypertensive eyes. Arch Ophthalmol. 1997;115:1137-1141.

7. Herman DC, Hodge DO, Bourne WM. Increased corneal thickness in patients with ocular hypertension. Arch Ophthalmol. 2001;119(3): 334-336.

8. Gordon MO, Beiser JA, Brandt JD, et al. The Ocular Hypertension Treatment Study: baseline factors that predict the onset of primary open-angle glaucoma. Arch Ophthalmol. 2002;120:714-720; discussion 829-830.

9. Leske MR, Hafez AS, Descovits D. Relationship between central corneal thickness and changes of optic nerve head topography and blood flow after intraocular pressure reduction in open-angle glaucoma and ocular hypertension. Arch Opthalmol. 2006;124(11):1568-1572.

10. Pakravan M, Parsa A, Sanagou M, et al. Central corneal thickness and correlation to disc size: a potential link to susceptibility to glaucoma. Br J Ophthalmol. 2007;91(1):26-28.

11. Toh T, Liew SH, Mac Kinnon JR, et al. Central corneal thickness is highly heritable: the twin eye study. Invest Opthalmol Vis Sci. 2005;46(10):3718-3722.

12. Aghaian E, Choe JE, Lin S, et al. Central corneal thickness of Caucasians, Chinese, Hispanic, Filipinos, African Americans, and Japanese in a glaucoma clinic. Opthalmology. 2004;111:2211-2219.

13. Bechmann M, Thiel MG, Roesen B, et al. Central corneal thicknessdetermined with optical coherence tomography in various types of glaucoma. Br J Opthalmol. 2000;84:688-691.

14. Wolfs RC, Klaver CC, Vingerling JR, et al. Distribution of central corneal thickness and its association with intraocular pressure. Am J Opthalmol. 1997;123:767-772.

15. Gorezis S, Gartzios C, Stefaniotou M, et al. Comparative results of central corneal thickness measurements in primary open-angle glaucoma, pseudoexfoliation glaucoma, and ocular hypertension. Ophthalmic Surg Lasers Imaging. 2008;39(1):17-21.

16. Ventura ACS, Bohnke M, Mojon DS. Central corneal thickness measurements in patients with normal tension glaucoma, primary open angle glaucoma, pseudoexfoliation glaucoma, or ocular hypertension. Br J Ophthalmol. 2001;85:792-795.

17. Lee ES, Kim CY, Ha SJ, et al. Central corneal thickness of Korean patients with glaucoma. Ophthalmology. 2007;114(5):927-930.

18. Jonas JB, Holbach L. Central corneal thickness and thickness of the lamina cribrosa in human eyes. Invest Opthalmol Vis Sci. 2005;46(4):1275-1279.

Clinical Ophthalmology

\section{Publish your work in this journal}

Clinical Ophthalmology is an international, peer-reviewed journal covering all subspecialties within ophthalmology. Key topics include: Optometry; Visual science; Pharmacology and drug therapy in eye diseases; Basic Sciences; Primary and Secondary eye care; Patient Safety and Quality of Care Improvements. This journal is indexed on Submit your manuscript here: http://www.dovepress.com/clinical-ophthalmology-journal
19. Copt RP, Thomas R, Mermoud A. Corneal thickness in ocular hypertension, primary open-angle glaucoma, and normal tension glaucoma. Arch Opthalmol. 1999;117:14-16.

20. Grodum K, Heijl A, Bengtsson B. Risk of glaucoma in ocular hypertension with and without pseudoexfoliation. Ophthalmology. 2005;112(3):386-390.

21. Inoue K, Okugawa K, Oshika T, et al. Morphological study of corneal endothelium and corneal thickness in pseudoexfoliation syndrome. Jpn J Opthalmol. 2003;47:235-239.

22. Yagci R, Eksioglu U, Midillioglu I, et al. Central corneal thickness in primary open angle glaucoma, pseudoexfoliative glaucoma, ocular hypertension, and normal population. Eur J Ophthalmol. 2005;15(3):324-328.

23. Dohadwala AA, Munger R, Damji KF. Positive correlation between Tono-Pen intraocular pressure and central corneal thickness. Ophthalmology. 1998;105(10):1849-1854.

24. Brandt JD, Beiser JA, Kass MA, et al. Central corneal thickness in the Ocular Hypertension Treatment Study (OHTS). Ophthalmology. 2001;108(10):1779-1788.

25. Kniestedt $\mathrm{C}$, Lin S, Choe J, et al. Correlation between intraocular pressure, central corneal thickness, stage of glaucoma, and demographic patient data: prospective analysis of biophysical parameters in tertiary glaucoma practice populations. J Glaucoma. 2006;15(2):91-97.

26. Herndon LW, Weizer JS, Stinnett SS. Central corneal thickness as a risk factor for advanced glaucoma damage. Arch Ophthalmol. 2004;122(1):17-21.

27. Papadia M, Sofianos C, Iester M, et al. Corneal thickness and visual field damage in glaucoma patients. Eye. 2007;21(7):943-947.

28. Chauhan BC, Hutchison DM, LeBlanc RP, et al. Central corneal thickness and progression of the visual field and optic disc in glaucoma. Br J Ophthalmol. 2005;89(8):1008-1012.

29. Sullivan-Mee M, Halverson KD, Saxon MC, et al. Central corneal thickness and normal tension glaucoma: a cross-sectional study. Optometry. 2006;77(3):134-140.

30. Hepsen IF, Yagci R, Keskin U. Corneal curvature and central corneal thickness in eyes with pseudoexfoliation syndrome. Can J Ophthalmol. 2007;42(5):677-680.

31. Arcieri ES, Pierre Filho PT, Wakamatsu TH, et al. The effects of prostaglandin analogues on the blood aqueous barrier and corneal thickness of phakic patients with primary open-angle glaucoma and ocular hypertension. Eye. 2008;22(2):179-183.

32. Sen E, Nalcacioglu P, Yazici A, et al. Comparison of the effects of latanoprost and bimatoprost on central corneal thickness. J Glaucoma. 2008;17(5):398-402.

33. Wilkerson M, Cyrlin M, Lippa EA, et al. Four-week safety and efficacy study of dorzolamide, a novel, active topical carbonic anhydrase inhibitor. Arch Ophthalmol. 1993;111(10):1343-1350.

34. Detorakis ET, Koukoula S, Chrisohoou F, et al. Central corneal mechanical sensitivity in pseudoexfoliation syndrome. Cornea. 2005;24(6):688-691.

35. Arnarsson A, Damji KF, Sverrisson T, et al. Pseudoexfoliation in the Reykjavik Eye Study: prevalence and related ophthalmological variables. Acta Ophthalmol Scand. 2007;85(8):822-827.

36. Puska P, Vassara K, Harju M, et al. Corneal thickness and corneal endothelium in normotensive subjects with unilateral pseudoexfoliation syndrome. Graefes Arch Clin Exp Opthalmol. 2000;238:659-663.

PubMed Central and CAS, and is the official journal of The Society of Clinical Ophthalmology (SCO). The manuscript management system is completely online and includes a very quick and fair peer-review system, which is all easy to use. Visit http://www.dovepress.com/ testimonials.php to read real quotes from published authors. 